\title{
OpenMP Teaching-Learning Based Optimization Algorithm over Multi-Core System
}

\author{
A. J. Umbarkar \\ Department of Information Technology, Walchand College of Engineering Sangli, MS, India \\ E-mail: anantumbarkar@ rediffmail.com \\ N. M. Rothe \\ Department of Computer Engineering, Walchand College of Engineering Sangli, MS, India \\ E-mail: nmrothe.11189@gmail.com \\ A.S. Sathe \\ Department of Information Technology, Walchand College of Engineering Sangli, MS, India \\ E-mail: ashutosh_sathe@gmail.com
}

\begin{abstract}
The problem with metaheuristics, including Teaching-Learning-Based Optimization (TLBO) is that, it increases in the number of dimensions (D) leads to increase in the search space which increases the amount of time required to find an optimal solution (delay in convergence). Nowadays, multi-core systems are getting cheaper and more common. To solve the above large dimensionality problem, implementation of TLBO on a multi-core system using OpenMP API's with $\mathrm{C} / \mathrm{C}++$ is proposed in this paper. The functionality of a multicore system is exploited using OpenMP which maximizes the CPU (Central Processing Unit) utilization, which was not considered till now. The experimental results are compared with a sequential implementation of Simple TLBO (STLBO) with Parallel implementation of STLBO i.e. OpenMP TLBO, on the basis of total run time for standard benchmark problems by studying the effect of parameters, viz. population size, number of cores, dimension size, and problems of differing complexities. Linear speedup is observed by proposed OpenMP TLBO implementation over STLBO.
\end{abstract}

Index Terms- Metaheuristic, Open Multiprocessing (OpenMP), Teaching-Learning-Based Optimization (TLBO), Unconstrained Function Optimization, Multicore.

\section{INTRODUCTION}

Optimization, in simple terms, means minimize the cost incurred and maximize the profit such as resource utilization. EAs are population based metaheuristic (means optimize problem by iteratively trying to improve the solution with regards to the given measure of quality) optimization algorithms that often perform well on approximating solutions to all types of problem because they do not make any assumptions about the underlying evaluation of the fitness function. There are many EAs available viz. Genetic Algorithm (GA) [1] , Artificial Immune Algorithm (AIA) [2], Ant Colony Optimization (ACO) [3], Particle Swarm Optimization (PSO) [4], Differential Evolution (DE) [5], [6], Harmony Search (HS) [7], Bacteria Foraging Optimization (BFO) [8], Shuffled Frog Leaping (SFL) [9], Artificial Bee Colony (ABC) $[10,11]$, Biogeography-Based Optimization (BBO)
[12], Gravitational Search Algorithm (GSA) [13], Grenade Explosion Method (GEM) [14] Firefly (FF) [15], [16] etc. To use any EA, a model of decision problem need to be built that specifies the decision variables, objective and constraints. These 3 parameters are necessary while building an optimization model. The solver will find values for the decision variables that satisfy the constraints while optimizing (maximizing or minimizing) the objective. But the problem with all the above EAs is that, to get an optimal solution, besides the necessary parameters. Various algorithms are parameter specific and that parameters need to be handled separately. For example, in case of GA, adjustment of the algorithm-specific parameters such as crossover rate, mutation rate, crossover type, type of encoding etc. affects the optimal solution obtained. Thus, in all the above EAs, selection of algorithm-specific parameter's value affects the optimal solution obtained. But in TLBO, there are no algorithm-specific parameters.

TLBO has been an efficient metaheuristic technique recently developed, for solving optimization problems with less computational effort and high consistency. Its advantage over other EAs is that, it has no algorithmspecific parameter (parameter less). The TLBO algorithm, which was proposed by Rao (2011), is actually based on the philosophy of teaching and learning. In a classroom, the teacher is considered as a highly learned person who tries to improve the outcome of learners by sharing his/her knowledge with them. It is called the Teacher phase of TLBO. Also, learners share and learn from the interaction among them, which help to improve their outcome. It is referred to as the Learner phase of TLBO [17]. In the entire process, TLBO tries to shift mean towards best.

Nowadays, multi-core CPUs (Central Processing Unit) are getting more and more common and cheaper. One cannot ignore their importance anymore. With the recent advancement of multi-core system, researchers have been modifying EAs for parallel implementation on a multicore system. The multi-core systems can run multiple 
instructions simultaneously on multiple cores; it increases overall speed for programs [18].

While using multi-core system, the improvement in performance depends upon the algorithms used and their implementation. In particular, possible benefits depend upon (or limited by) the fraction of the algorithm that can be managed in parallel on multiple cores simultaneously; this issue is explained in Amdahl's law. The problems which are embarrassingly parallel may realize speedup factors near to the number of cores. Even more speedup factors can be realized by splitting up the problem so that it can fit within the cache of each core(s), thereby avoiding use of much slower main memory of the system. In order to accelerate performance of application, programmers have to work more on re-factoring the whole problem. The parallelization of algorithms on a multi-core the system is a significant ongoing topic of research.

Recent advancement in High Performance Computing (HPC) have seen the usage of many cores of multi-core system (viz. i3, i5, i7 etc.) for solving computationally intensive task parallelly. OpenMP is an API for writing shared-memory parallel applications in $\mathrm{C}, \mathrm{C}++$, and FORTRAN. With the release of API specifications (in 1997) by the OpenMP Architecture Review Board (ARB), use of CPU's computational power (in particular, multiple cores of multi-core CPU) for parallel computing has become easy [19]. OpenMP is ideally suited for multi-core architectures hence it allows programs to be parallelized incrementally with little programming efforts.

The designers of OpenMP developed a set of compiler pragmas, directives, and environment variables, function calls which are platform-independent. In application exactly where and how to insert threads are explicitly instructed by these constructs. Most of the loops can be parallelized just by adding \#pragma_omp_parallel_for before the 'for loop' construct in $\mathrm{C}$. The main tasks while using OpenMP are 1) to determine which loops should be threaded and 2) to restructure the algorithms for maximum performance. The OpenMP provides maximum performance, if it is applied to threads in the application which are most time-consuming loops.

Thus, if the bottlenecks in the algorithm are identified and modified suitably, using OpenMP, one can easily exploit the functionality of multi-core system and can maximize the utilization of all the cores of multi-core system which is necessary from the optimization point of view (for example, maximize the resource utilization). This paper contributes towards this direction and undertakes a detailed study by investigating the effect of number of cores, dimension size, population size, and problem complexity on the speedup of TLBO algorithm. In the remainder of this paper, we give a brief literature review of TLBO and its applications. Thereafter, we discuss the possibilities of tweaking a TLBO to make it suitable for parallel implementation on a multi-core system. Then, we present results on a few test problems of different complexities and show appreciable speed-ups using our proposed algorithm.

\section{LITERATURE REVIEW}

TLBO is very effective than other metaheuristics because of its characteristics viz. parameter less, high consistency, and less computation effort. It outperforms some of the well-known metaheuristics regarding constrained benchmark functions, constrained mechanical design [20], and continuous non-linear numerical optimization problems [17], it is being used by various researchers as a replacement for the other EAs available. Such a breakthrough has steered $\mathrm{C}^{\sim}$ repinšek et al. [21] towards investigating the secrets of TLBO's dominance. They investigated some mistakes regarding TLBO through code-reviews and experiments respectively. However, Waghmare in [22] commented on the work of $\mathrm{C}^{\sim}$ repinšek et al. He not only addressed the queries raised by $\mathrm{C}^{\sim}$ repinšek et al. but also re-examined the experimental results, which demonstrates that the TLBO algorithm performs well on the problems where the fitness-distance correlations are low by proper tuning of the common control parameters of the algorithm, and corrected the understanding about the TLBO algorithm in an objective manner. TLBO has been used by number of researchers to solve their problems and found it effective than other Metaheuristic. Krishnanand et al. in [23] have applied a multi-objective TLBO algorithm with nondomination based sorting to solve the environmental or economic dispatch (EED) problem containing the incommensurable objectives of best economic dispatch and least emission dispatch. Rao and Patel in [24] explored the use of TLBO and ABC algorithms for determining the optimum operating conditions of combined Brayton and inverse Brayton cycles. Rao et al. in [25] proposed the optimization of mechanical design problems using TLBO and tested it on five different constrained benchmark test functions with different characteristics, four different benchmark mechanical design problems and six mechanical design optimization problems. González-Álvarez et al. in [26] proposed Multi-objective TLBO (MO-TLBO) for solving Motif Discovery Problem (MDP) and solved a set of twelve biological instances belonging to different organisms. Rao and Patel introduced and investigated the effect of elitism on the performance of TLBO algorithm while solving complex constrained optimization problems [27] and unconstrained benchmark problems [28]. Population size and Number of generation, these parameter affects the performance of TLBO are also investigated. Rao and Kalyankar in [29] made an attempt to achieve conflicting objectives by finding optimum parameter settings for the LBW process by applying TLBO for parameter optimization of the LBW process. Rajasekhar et al. in [30] proposed a new variant of TLBO, termed as Elitist Teaching-Learning Opposition based (ETLOBA) Algorithm for numerical function optimization, which is empowered with two mechanisms, one is elitism and second is Opposition method (helps to improve the capability of searching), to reach the accurate global optimum with less time complexity. Rao and Patel in [31] introduced a modified version of the TLBO algorithm and applied the same for the multi-objective optimization 
of heat exchangers. Pawar and Rao in [32] presented TLBO to find the optimal combination of process parameters of the abrasive water jet, grinding and milling machining processes. Rao and Kalyankar in [33] applied TLBO for the process parameter optimization of electrochemical machining (ECM) process and electrochemical discharge machining (ECDM) process. Rao et al. in [34] proposed TLBO to solve continuous unconstrained and constrained optimization problems. Niknam et al. in [35] proposed a $\theta$-multi-objective-TLBO algorithm to solve the dynamic economic emission dispatch problem.

\section{ALGORITHM}

Most of the times TLBO uses only single core of multi-core system thereby leaving other cores idle. One of the goals of optimization is to maximize the profit such as resource utilization, so it's necessary to make algorithm parallel either by design or programming way, which exploits all the cores of multi-core system and maximize the CPU utilization. OpenMP maps threads onto physical cores of CPU, hence all the OpenMP threads run in parallel and provide more optimal solution in less amount of time thereby providing speedup compare to Simple TLBO (STLBO). The basic TLBO algorithm can be stated as in fig. 1 .

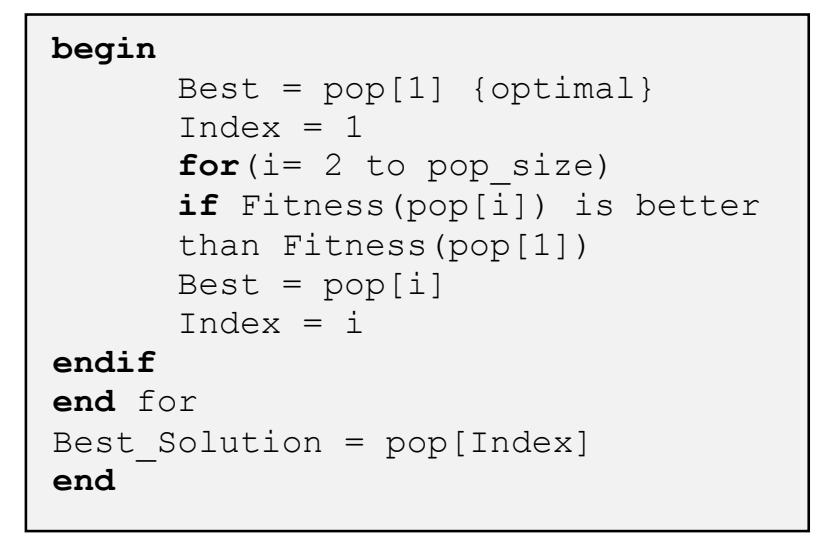

Fig. 1. Pseudo code of TLBO

The OpenMP algorithm exactly emulates the sequential algorithm where calculation of Fitness, calculation of mean, calculation of best, and comparison of fitness function remains same whereas small changes are introduced to achieve better result/performance.

In the following subsections, how the different functions of TLBO work, how we have parallelized them, and discuss the intricacies involved in are mentioned in the paper.

\section{A. Initialize_Population}

This method reads the basic TLBO parameters, including population and generation sizes, types of decision variables and their lower and upper limits. This remains unchanged in the parallel algorithm. Thus, this method generates an initial population (set of possible candidate solutions) randomly by taking population size, number of decision variables, and upper $\&$ lower limit of decision variables into consideration. This population generated serves as an input to Teacher-Phase of TLBO algorithm.

\section{B. Calculate_Fitness}

This method evaluates fitness of each individual of population using the defined objective function, which indicates how much each variable contributes to the value to be optimized (either maximize or minimize) in the problem. The fitness evaluation of each individual is independent step; an OpenMP parallel pragma is used to create multiple threads, ranging from 2 to 32, to evaluate each individual's fitness separately and are mapped onto corresponding numbers of physical cores (e.g. 2 threads are mapped onto 2 physical cores. Similarly, 4, 8, 16 and 32 threads are mapped onto 4, 8, 16 and 32 physical cores respectively). It is possible to map more than one thread on a core but in practice it is best to have one-to-one mapping. In this way, this step is parallelized and reduced the overall time required to evaluate the fitness of all individuals of the population and also maximized the CPU utilization (by utilizing all the cores of the CPU). This method is called twice, once in Teacher phase and once in Learner phase (in case of elitist TLBO it is called more than twice), parallelization of this method helps significantly in reducing the amount of time required for total execution.

\section{Calculate_Mean_Vector}

This method calculates the mean of each decision variable in the population. This method is parallelized by creating multiple OpenMP threads (2 to 32 ) which are deployed over the multiple cores (2 to 32 ) of CPU (oneto-one mapping). Thus by calculating means of each decision variable simultaneously, the amount of time required to calculate mean of all decision variables is reduced and this simultaneous execution maximizes the CPU utilization as well.

\section{Best_Solution}

This method finds the best solution from the population based on its fitness value. An individual candidate solution having optimum fitness value (either minimum or maximum, as per the requirement) is termed as Best_Solution (or Teacher) and is selected to calculate new population in Teacher-phase. The simple logic applied for this method is in fig. 2 .

To parallelize this method, REDUCTION (Min or Max) pragma of OpenMP can be used, but it is found that, the use of REDUCTION pragma deteriorates the performance of this method, because to select the Best_Solution, first, we need to find the optimal fitness value and its index and based on that index the candidate solution from the population is selected. This calculation of index adds overhead which deteriorates the performance. So, above simple logic is applied to avoid the reduction in performance. 


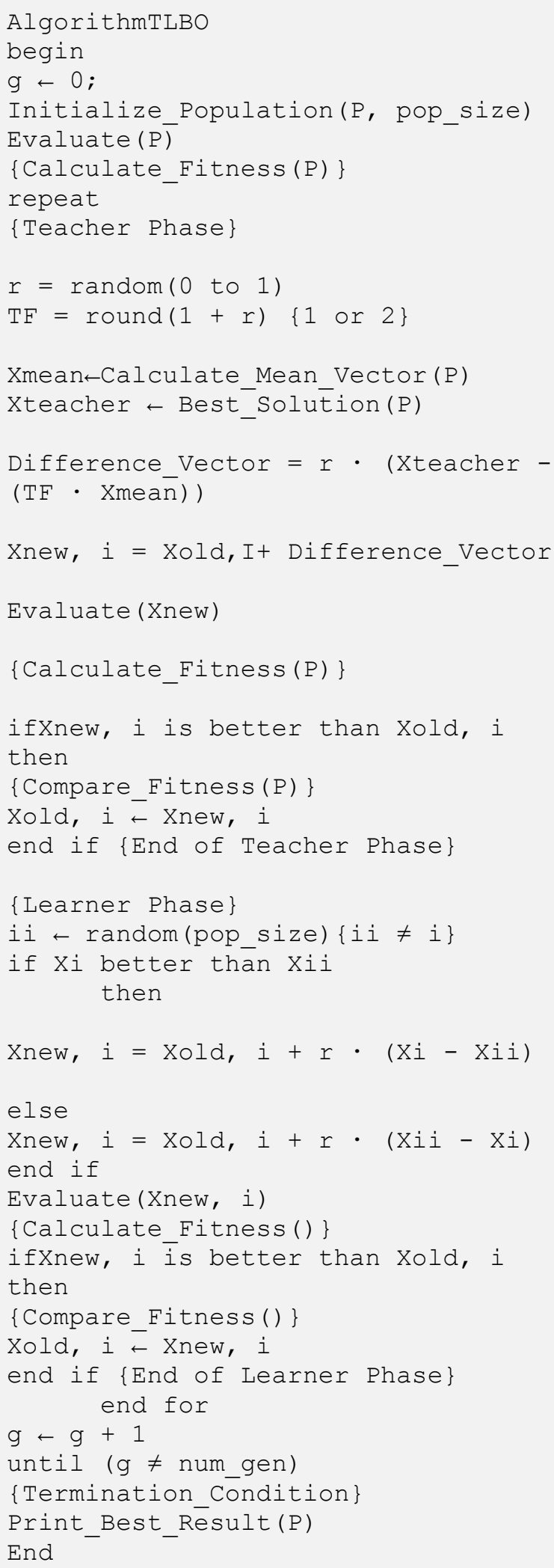

Fig. 2. Pseudo code of Best Solution

\section{E. Create_New_Population}

Based on the values of Mean, Best_Solution, $r$ and $\mathrm{T}_{\mathrm{F}}$ ( $\mathrm{r}$ and $\mathrm{T}_{\mathrm{F}}$ are randomly generated), a Difference_vector is calculated and added to each individual solution of the old population to get the new population. This addition of Difference_Vector to each individual is an independent step; an OpenMP parallel pragma is used to create multiple threads (2 to 32 ) for performing the addition separately and are mapped onto multiple physical cores (2 to 32). Thus, by adding the Difference_Vector to each candidate solution simultaneously, the amount of time required to create new population is decreased significantly and this simultaneous creation maximizes the CPU utilization. After this, the fitness value of the new population is evaluated.

\section{F.Compare_Fitness}

This method compares the fitness of newly calculated population with the fitness of the old population. The fitness of each candidate solution of the new population is compared with the fitness of the corresponding candidate solution of the old population and the one with the best fitness value is selected (survival of the fittest). This oneto-one comparison is also an independent step and thus, parallelized by creating multiple OpenMP threads ( 2 to $32)$, which are mapped on multiple cores (2 to 32 ) of CPU. This method is also called twice, once in Teacher phase and once in Learner phase. Hence, the parallelization of this step significantly reduced the overall time required to compare the fitness of the new population with the old population and also optimize the CPU utilization.

\section{TEST FUNCTION}

In the field of optimization, it is a common practice to compare different algorithms using different benchmark problems. In this work also different benchmark problems are considered having different characteristics such as separability, multimodality, and regularity. If the function has two or more local optima then it is considered as a multimodal function. A separable function can be written as a sum of functions of variables separately. A function is regular if it is differentiable at each point of its domain. It is very difficult to optimize non-separable functions and difficulty increases if the function is multimodal. Complexity increases when the local optima are randomly distributed. Moreover, complexity increases with the increase in the dimensionality. The benchmark problems used for experimentation are shown in table 1 .

\section{EXPERIMENT RESULT}

To validate Parallel implementation of STLBO (i.e. OpenMP TLBO) results are compared with the results of sequential STLBO algorithms for different benchmark problems existing in the literatures. Paper focuses on showing the speedup of programming parallel algorithm. The objective functions considered for experimentation are to check the algorithm's ability to find the optimal solution. 
Table 1. List of test problems

\begin{tabular}{|c|c|c|c|c|c|}
\hline Sr. No. & Function Name & Equation & Range & $\mathbf{F}(\mathbf{x})$ & $\mathrm{C}$ \\
\hline $\mathrm{F} 1$ & De Jong & $f(x)=\sum_{i=1}^{n} x_{i}^{2}$ & {$[-5.12,5.12]$} & 0 & US \\
\hline F2 & $\begin{array}{c}\text { Axis Parallel } \\
\text { Hyper-Ellipsoid }\end{array}$ & $f(x)=\sum_{i=1}^{n}\left(i \cdot x_{i}^{2}\right)$ & {$[-5.12,5.12]$} & 0 & US \\
\hline F3 & $\begin{array}{c}\text { Sum of } \\
\text { Different Power }\end{array}$ & $f(x)=\sum_{i=1}^{n}\left|x_{i}\right|^{i+1}$ & {$[-1,1]$} & 0 & US \\
\hline F4 & Quartic & $f(x)=\sum_{i=1}^{n} i x_{i}^{4}+\operatorname{random}[0,1)$ & {$[-1.28,1.28]$} & 0 & US \\
\hline F5 & SumSquares & $f(x)=\sum_{i=1}^{n} i x_{i}^{2}$ & {$[-10,10]$} & 0 & US \\
\hline F6 & Zakharov & $f(x)=\sum_{i=1}^{n} x_{i}^{2}+\left(\sum_{i=1}^{n} 0.5 i x_{i}\right)^{2}+\left(\sum_{i=1}^{n} 0.5 i x_{i}\right)^{4}$ & {$[-5,10]$} & 0 & UN \\
\hline F7 & Hper Sphere & $f(x)=\sum_{i=1}^{6} x_{i}^{2}$ & {$[-5.12,5.12]$} & 0 & UN \\
\hline F8 & Rastrigin & $f(x)=\sum_{i=1}^{n}\left[x_{i}^{2}-10 \cos \left(2 \pi x_{i}\right)+10\right]$ & {$[-5.12,5.12]$} & 0 & MS \\
\hline F9 & Ackley & $f(x)=-20 \exp \left(-0.2 \sqrt{\frac{1}{n}} \sum_{i=1}^{n} x_{i}^{2}\right)-\exp \left(\frac{1}{n} \sum_{i=1}^{n} \cos \left(2 \pi x_{i}\right)\right)+20+2.718$ & {$[-32.768,32.768]$} & 0 & MN \\
\hline F10 & Griewangk & $f(x)=\frac{1}{4000} \sum_{i=1}^{n} x_{i}^{2}-\prod_{i=1}^{n} \cos \left(\frac{x_{i}}{\sqrt{i}}\right)+1$ & {$[-600,600]$} & 0 & MN \\
\hline
\end{tabular}

In the experimentation following system configuration is used - Intel(R) Core i7-2600 CPU $3.40 \mathrm{GHz}$ processor with RAM of $4.00 \mathrm{~GB}$ (3.16 usable) (8-cores). Results are taken for 1-core STLBO, 2-cores OpenMP TLBO, 4cores OpenMP TLBO and 8-cores OpenMP TLBO on the above system by setting a task set which assigns a particular CPU cores to process or programs. To take the results of OpenMP TLBO on 16-cores and 32-cores, 8 socket Quad Core AMD Opteron ${ }^{\mathrm{TM}}$ Processor (Model 8378, $2.4 \mathrm{GHz}, 75 \mathrm{~W}$ ACP), RAM 24 GB is used.

In the experimentation, values of $\mathrm{Pn}$ and $\mathrm{Dn}$ are taken very large i.e. $\mathrm{Pn}=1000$ and $\mathrm{Dn}=5000$, and OSF is taken as a criterion for terminating the execution of the algorithm. In case of OSF, an algorithm is considered to be successful if the difference between the optimum solution found by the algorithm and the global optimum value is less than 0.001 .

\section{A. Convergence of Proposed OpenMP TLBO Algorithms}

Programming parallelization of algorithm ensure that the solution achieved should be similar in quality in compassion serial implementation. Table 2 demonstrates the ability of the proposed algorithm to find the optimal solution on 2, 4, 8, 16 and 32 cores. Population size and dimensions are set to 1000 and 200 respectively. OpenMP TLBO algorithm converges early and produces the same and better OSF than STLBO algorithm.

Table 2. Optimum Solution Found (OSF) on OpenMP TLBO algorithm

\begin{tabular}{|c|c|c|c|c|c|c|}
\hline \multirow{2}{*}{ Function } & STLBO & \multicolumn{5}{|c|}{ OSF in OpenMP TLBO Cores } \\
\cline { 2 - 7 } & OSF & $\mathbf{2}$ & $\mathbf{4}$ & $\mathbf{8}$ & $\mathbf{1 6}$ & $\mathbf{3 2}$ \\
\hline F1 & 0.0008 & 0.0007 & 0.0008 & 0.0008 & 0.0008 & 0.0008 \\
\hline F2 & 0.0008 & 0.0008 & 0.0009 & 0.0008 & 0.0008 & 0.0007 \\
\hline F3 & 0.0006 & 0.0006 & 0.0005 & 0.0006 & 0.0005 & 0.0004 \\
\hline F4 & 0.0008 & 0.0007 & 0.0008 & 0.0008 & 0.0008 & 0.0008 \\
\hline F5 & 0.0008 & 0.0007 & 0.0007 & 0.0008 & 0.0008 & 0.0008 \\
\hline F6 & 0.0008 & 0.0007 & 0.0008 & 0.0008 & 0.0007 & 0.0009 \\
\hline F7 & 0.0008 & 0.0008 & 0.0007 & 0.0008 & 0.0008 & 0.0009 \\
\hline F8 & 0.0009 & 0.0008 & 0.0008 & 0.0008 & 0.0009 & 0.0008 \\
\hline F9 & 0.0009 & 0.0009 & 0.0009 & 0.0009 & 0.0009 & 0.0009 \\
\hline F10 & 0.0008 & 0.0008 & 0.0009 & 0.0008 & 0.0008 & 0.0008 \\
\hline
\end{tabular}




\section{B. Effect of Number of Cores}

Execution times of OpenMP TLBO on 2 to 32 cores demonstrate the speedup achieved. The speedup value is calculated by dividing the overall computational time of sequential implementation STLBO by the time taken by the parallel implementation of STLBO (i.e. OpenMP
TLBO). It is seen that, as the number of cores increases, speedup increases. The results of speedup are shown in table $3 \mathrm{~A}$ and table $3 \mathrm{~B}$ on 2 to 32 cores. Fig. 3 shows speedup achieved for an average of all functions (F1 to F10) on 2 to 32 cores.

Table 3A. Speed ups obtained by OpenMP TLBO algorithm on 2, 4, and 8 cores system

\begin{tabular}{|c|c|c|c|c|c|c|c|}
\hline \multirow{2}{*}{ Function } & STLBO & \multicolumn{2}{|c|}{ OpenMP TLBO on 2-cores } & \multicolumn{2}{c|}{ OpenMP TLBO on 4-cores } & \multicolumn{2}{c|}{ OpenMP TLBO on 8-cores } \\
\cline { 2 - 8 } & Time & Time & Speedup & Time & Speedup & Time & Speedup \\
\hline F1 & 20.0599 & 12.25039 & 1.637490725 & 6.80687 & 2.947007949 & 6.388372 & 3.14006448 \\
\hline F2 & 28.76988 & 15.71026 & 1.831279686 & 9.38783 & 3.064593202 & 8.916324 & 3.226652598 \\
\hline F3 & 11.317109 & 5.625516 & 2.011745945 & 3.288464 & 3.441457471 & 1.957506 & 5.78139173 \\
\hline F4 & 61.56627 & 29.45228 & 2.090373648 & 16.5931 & 3.710353701 & 15.70955 & 3.9190346 \\
\hline F5 & 30.47906 & 16.24502 & 1.876209448 & 9.305884 & 3.27524607 & 8.906804 & 3.421997385 \\
\hline F6 & 29.30387 & 15.63251 & 1.874546698 & 9.050192 & 3.237927991 & 9.121547 & 3.212598696 \\
\hline F7 & 19.76654 & 10.8366 & 1.82405367 & 6.653588 & 2.970809133 & 5.648631 & 3.499350551 \\
\hline F8 & 49.36975 & 26.42954 & 1.867976136 & 14.81388 & 3.332668416 & 11.52902 & 4.282215661 \\
\hline F9 & 48.05734 & 25.6792 & 1.871450045 & 14.3507 & 3.348780199 & 10.58446 & 4.540367671 \\
\hline F10 & 53.6774 & 29.20771 & 1.837781873 & 15.82984 & 3.390899719 & 11.83986 & 4.533617796 \\
\hline
\end{tabular}

Table 3B. Speed ups obtained by OpenMP TLBO algorithm on 16 and 32 cores system

\begin{tabular}{|c|c|c|c|c|}
\hline \multirow{2}{*}{ Function } & \multicolumn{2}{|c|}{ OpenMP TLBO on 16-cores } & \multicolumn{2}{c|}{ OpenMP TLBO on 32-cores } \\
\cline { 2 - 5 } & Time & Speedup & Time & Speedup \\
\hline F1 & 5.077111 & 3.951046176 & 4.406814 & 4.55201876 \\
\hline F2 & 6.983889 & 4.119464098 & 6.178805 & 4.656220742 \\
\hline F3 & 1.619893 & 6.986331196 & 1.392834 & 8.125238901 \\
\hline F4 & 13.5134 & 4.55594225 & 11.22061 & 5.486891533 \\
\hline F5 & 7.308339 & 4.170449674 & 5.678492 & 5.367456712 \\
\hline F6 & 6.979426 & 4.19860745 & 5.337963 & 5.489710213 \\
\hline F7 & 4.948327 & 3.994590495 & 3.862162 & 5.117998675 \\
\hline F8 & 8.550941 & 5.773604332 & 6.048994 & 8.161646383 \\
\hline F9 & 8.618475 & 5.576083936 & 6.007755 & 7.999217678 \\
\hline F10 & 8.781576 & 6.112501902 & 6.028889 & 8.903365114 \\
\hline
\end{tabular}

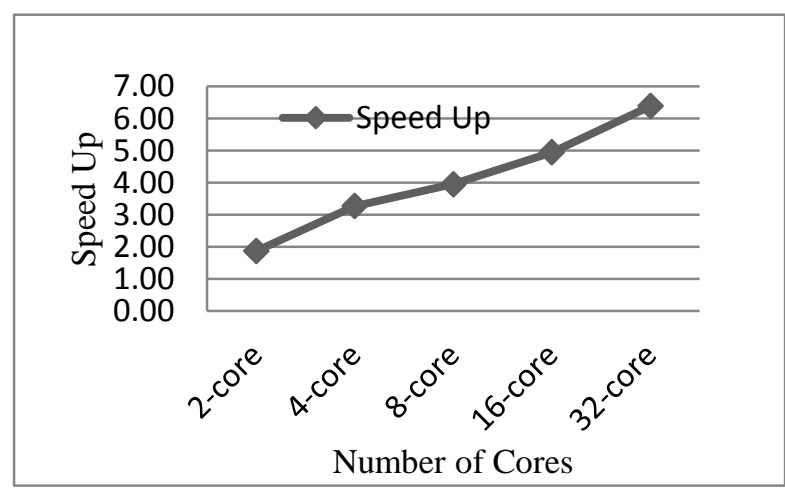

Fig. 3. Speedup versus number of cores

\section{Optimum Utilization of Computing Resource}

Fig. 4 shows the utilization of multi-core system by STLBO algorithm. Fig. 5 shows the utilization of multicore system by OpenMP TLBO. These results show the significant speedup, optimum utilization of multi-core systems.

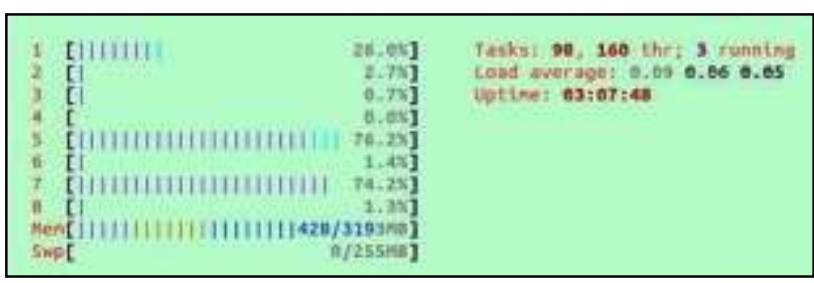

Fig. 4. Utilization of multi-core (8-cores) system by STLBO algorithm 


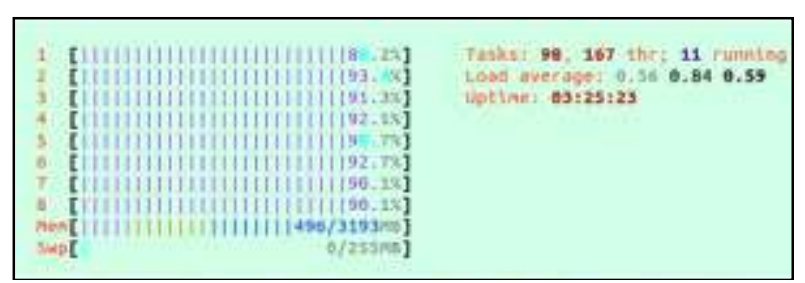

Fig. 5. Utilization of multi-core (8-cores) system by OpenMP TLBO algorithm

\section{Effect of Problem and EA Parameters}

Apply parallel implementation (OpenMP TLBO) to all the test problems shown in Table 1 for studying the effect of the following properties on the speedup of the algorithm:

1) Number of design variables
2) Population size

3) Problem complexity

This experimentation is taken on Intel Core i7-2600 CPU (8-cores CPU system). Each run is terminated after the OSF and normalized over 10 runs.

Table 4 show the results experimentation for speedup and CPU utilization on 8-core system for dimension 200. It shows that minimum speedup is 2.97 and maximum speedup is 4.67. CPU utilization increases up to $93 \%$ using OpenMP TLBO algorithm as compare to STLBO. Table 5 show the results experimentation for speedup and CPU utilization on 8-core system for dimension 2000. It shows that minimum speedup is 3.38 and maximum speedup is 5.21. CPU utilization increases up to $93 \%$ using OpenMP TLBO algorithm as compare to STLBO.

Table 4. Performance of STLBO and OpenMP TLBO on 8-cores system for Pn=1000 and Dn=200.

\begin{tabular}{|c|c|c|c|c|c|c|}
\hline Type & $\begin{array}{c}\text { STLBO } \\
\text { Time }\end{array}$ & $\begin{array}{c}\text { OpenMP } \\
\text { TLBO Time }\end{array}$ & Speedup & Efficiency & $\begin{array}{c}\text { STLBO CPU } \\
\text { Utilization }\end{array}$ & $\begin{array}{c}\text { OpenMP TLBO } \\
\text { CPU Utilization }\end{array}$ \\
\hline F1 & 0.5943917 & 0.1999474 & 2.972740331 & 37.15925413 & 22.11 & 89.88 \\
\hline F2 & 0.7966197 & 0.2425574 & 3.284252305 & 41.05315381 & 23.01 & 92.11 \\
\hline F3 & 0.10658835 & 0.03465242 & 3.07592803 & 38.44910038 & 25.1 & 92.33 \\
\hline F4 & 1.744723 & 0.5422093 & 3.217803531 & 40.22254414 & 24.31 & 92.31 \\
\hline F5 & 0.855884 & 0.2612472 & 3.276146118 & 40.95182647 & 23.74 & 90.11 \\
\hline F6 & 0.7974406 & 0.2474692 & 3.22238323 & 40.27979037 & 26.54 & 93.21 \\
\hline F7 & 0.586408 & 0.1817133 & 3.227105556 & 40.33881945 & 23.54 & 93.24 \\
\hline F8 & 1.870083 & 0.4234461 & 4.416342481 & 55.20428102 & 28.47 & 90.12 \\
\hline F9 & 1.872172 & 0.4246587 & 4.408650994 & 55.10813743 & 24.56 & 90.21 \\
\hline F10 & 1.937671 & 0.414895 & 4.670268381 & 58.37835476 & 24.65 & 90.12 \\
\hline
\end{tabular}

Table 5. Performance of STLBO and OpenMP TLBO on 8-cores system for Pn=1000 and Dn=2000.

\begin{tabular}{|c|c|c|c|c|c|c|}
\hline Type & $\begin{array}{c}\text { TLBO } \\
\text { Time }\end{array}$ & $\begin{array}{c}\text { OpenMP } \\
\text { TLBO Time }\end{array}$ & Speedup & Efficiency & $\begin{array}{c}\text { TLBO CPU } \\
\text { Utilization }\end{array}$ & $\begin{array}{c}\text { OpenMP TLBO } \\
\text { CPU Utilization }\end{array}$ \\
\hline F1 & 7.389233 & 2.182785 & 3.385231711 & 42.31539639 & 21.32 & 89.98 \\
\hline F2 & 10.71307 & 3.246257 & 3.300129965 & 41.25162456 & 29.54 & 91.24 \\
\hline F3 & 2.911842 & 0.5580366 & 5.218012582 & 65.22515727 & 24.51 & 89.62 \\
\hline F4 & 19.90186 & 6.123099 & 3.25029205 & 40.62865062 & 23.54 & 93.24 \\
\hline F5 & 11.27272 & 3.225995 & 3.494338956 & 43.67923695 & 24.56 & 90.21 \\
\hline F6 & 10.695324 & 3.287837 & 3.252997031 & 40.66246289 & 23.66 & 91.27 \\
\hline F7 & 7.442053 & 2.217287 & 3.356377862 & 41.95472327 & 28.87 & 92.22 \\
\hline F8 & 19.44875 & 4.591783 & 4.235555121 & 52.94443901 & 26.44 & 92.22 \\
\hline F9 & 19.07972 & 4.297528 & 4.439696495 & 55.49620619 & 20.12 & 90.11 \\
\hline F10 & 21.24441 & 4.552396 & 4.666643675 & 58.33304594 & 22.11 & 89.88 \\
\hline
\end{tabular}

Results clearly indicate that for larger population size and design variables size, the use of OpenMP implementations of existing STLBO on a multi-core platform is beneficial.

Efficiency of the proposed algorithm is calculated using (1).

Efficiency $=($ Speedup $/$ Number of cores $) * 100$

\section{CONCLUSION}

This paper experimented the programming parallel TLBO(OpenMP TLBO) on multi-core system for unconstrained optimization.

OpenMP TLBO is successful in utilizing all the cores of multicore system than the STLBO. For all function the OpenMP TLBO gives remarkable speedup over STLBO. 
The maximum utilization of multicore system helps OpenMP TLBO to converge early.

Results show that, the OpenMP TLBO gives linear speedup and optimizes the CPU utilization against the sequential implementation of TLBO (STLBO).

In future scope, researchers can experiment the proposed OpenMP TLBO on CEC 2013 function bed. Further constrained optimization test bed could be also experimented.

\section{ACKNOWLEDGEMENT}

The authors are thankful to the anonymous reviewers as well as the editors for their valuable comments and suggestions which have led to improve the quality of the paper. Our special thanks to Dr. R. V. Rao for their work on Teaching Learning Based Optimization Algorithm published in various journals and conferences.

\section{REFERENCES}

[1] J. Holland, Adaptation in Natural and Artificial Systems, University of Michigan Press, Ann Arbor, 1975.

[2] J. Farmer, et al., "The immune system, adaptation and machine learning," Physica D, vol. 2, pp.187-204, 1986. DOI: 10.1016/0167-2789(81)90072-5

[3] M. Dorigo, "Optimization, Learning and Natural Algorithms", Ph.D. thesis, Politecnico di Milano, Italy, 1992.

[4] J. Kennedy and R. Eberhart, "Particle swarm optimization," Proceedings of IEEE International Conference on Neural Networks, Piscataway, NJ, vol. 4, pp. 1942-1948, 1995. DOI: 10.1109/ICNN.1995.488968

[5] E. Mezura-Montes, et al., "Differential evolution in constrained numerical optimization: an empirical study," Information Sciences, vol. 180 pp. 4223-4262, 2010. DOI: 10.1016/j.ins.2010.07.023

[6] R. Storn and K. Price, "Differential evolution - a simple and efficient heuristic for global optimization over continuous spaces," Journal of Global Optimization, vol. 11 pp. 341-359, 1997. DOI: 10.1023/A:1008202821328

[7] Z. Geem, et al., "A new heuristic optimization algorithm: harmony search," Simulation, vol. 76, pp. 60-70, 2001. DOI: 10.1177/003754970107600201

[8] K. Passino, "Biomimicry of bacterial foraging for distributed optimization and control," IEEE Control Systems Magazine, vol. 22 pp. 52-67, 2002, DOI: 10.1109/MCS.2002.1004010

[9] M. Eusuff and E. Lansey, "Optimization of water distribution network design using the shuffled frog leaping algorithm," Journal of Water Resources Planning and Management, ASCE, vol. 129 pp. 210-225, 2003, [Online]. Available: DOI: http://dx.doi.org/10.1061/(ASCE)07339496(2003)129:3(210)

[10] B. Akay and D. Karaboga, "A modified artificial bee colony (ABC) algorithm for constrained optimization problems," Applied Soft Computing, vol.11, pp.3021-3031, 2011. DOI: 10.1016/j.asoc.2010.12.001

[11] D. Karaboga, "An Idea Based on Honey Bee Swarm for Numerical Optimization," Technical REPORT-TRO6, Erciyes University, Engineering Faculty, Computer Engineering Department, 2005
[12] D. Simon, "Biogeography-based optimization," IEEE Transactions on Evolutionary Computation, vol. 12 pp. 702-713, 2008. DOI: 10.1109/TEVC.2008.919004

[13] E. Rashedi, et al, "GSA: a gravitational search algorithm," Information Sciences, vol. 179, pp. 2232-2248, 2009. DOI: 10.1016/j.ins.2009.03.004

[14] A. Ahrari and A. Atai, "Grenade explosion method - a novel tool for optimization of multimodal functions," Applied Soft Computing, vol. 10, pp. 1132-1140, 2010. DOI: 10.1016/j.asoc.2009.11.032

[15] R. Benabid, et al, "Application of Firefly Algorithm for Optimal Directional Overcurrent Relays Coordination in the Presence of IFCL," International Journal of Intelligent Systems and Applications, Vol. 6, pp.44-53, 2014. DOI: 10.5815/ijisa.2014.02.06

[16] I. Fister, "A comprehensive review of firefly algorithms," Swarm and Evolutionary Computation, Vol. 13, pp. 34-46. DOI: 10.1016/j.swevo.2013.06.001

[17] R. V. Rao, et al, "Teaching-learning-based optimization: an optimization method for continuous non-linear large scale problems," Information Sciences, vol. 183 pp. 1-15, 2012. DOI: $10.1016 /$ j.ins.2011.08.006

[18] Suleman A. "What makes parallel programming hard," http://www.futurechips.org/tips-for-power-coders/parallel programming.html, 20 may 2011.

[19] http://openmp.org/wp/about-openmp/

[20] R.V Rao, et al, "Teaching-learning-based optimization: A novel method for constrained mechanical design optimization problems," Computer-Aided Design, vol. 43, pp. 303-315, 2011 DOI: 10.1016/j.cad.2010.12.015

[21] $\mathrm{C}^{\sim}$ repinšek $\mathrm{M}$, et al, "A note on teaching-learning-based optimization algorithm," Information Sciences, vol 212, pp. 79-93, 2012. DOI: 10.1016/j.ins.2012.05.009

[22] G. Wadhmare, "Comments on A note on teachinglearning-based optimization algorithm," Information Sciences, vol. 229, pp. 159-169, 2013. DOI: 10.1016/j.ins.2012.11.009

[23] K.R. Krishnanand, et al, "Application of Multi-Objective Teaching-Learning-Based Algorithm to an Economic Load Dispatch Problem with Incommensurable Objectives," SEMCCO, LNCS, vol. 7076, pp. 697-705, 2011. DOI: 10.1007/978-3-642-27172-4_82.

[24] R.V. Rao and V. Patel, "Multi-objective optimization of combined Brayton and inverse Brayton cycles using advanced optimization algorithms," Engineering Optimization, Vol. 44, pp. 965-983, 2012. DOI: 10.1080/0305215X.2011.624183.

[25] R.V. Rao, et al, "Teaching-learning-based optimization: A novel method for constrained mechanical design optimization problems," Computer-Aided Design, vol. 43, pp. 303-315, 2011. DOI: 10.1016/j.cad.2010.12.015

[26] D.L. González-Álvarez, et al, "Multiobjective TeachingLearning Based Optimization (MO-TLBO) for Motif Finding," 13th IEEE International Symposium on Computational Intelligence and Informatics, vol. 68, pp. 141-146, 2012. DOI: 10.1109/CINTI.2012.6496749

[27] R.V. Rao and V. Patel, "An elitist teaching-learning-based optimization algorithm for solving complex constrained optimization problems," International Journal of Industrial Engineering Computations 3, pp. 535-560, 2012. DOI: 10.5267/j.ijiec.2012.03.007

[28] R.V. Rao and V. Patel, "Comparative performance of an elitist teaching-learning-based optimization algorithm for solving unconstrained optimization problems," International Journal of Industrial Engineering Computations, vol. 4, pp. 29-50, 2013. DOI: 10.5267/j.ijiec.2012.09.001 
[29] R.V. Rao and V.D. Kalyankar, "Multi-objective multiparameter optimization of the industrial LBW process using a new optimization algorithm," Proceedings of the Institution of Mechanical Engineer sPart B: Journal of Engineering Manufacture, vol 226, pp. 1018-1025, 2012. DOI: $10.1177 / 0954405411435865$.

[30] A. Rajasekhar, et al, "Elitist Teaching Learning Opposition based Algorithm for Global Optimization," IEEE International Conference on Systems, Man, and Cybernetics, pp. 1124-1129, 2012. DOI: 10.1109/ICSMC.2012.6377882

[31] R.V. Rao and V. Patel, "Multi-objective optimization of heat exchangers using a modified teaching-learning-based optimization algorithm," Applied Mathematical Modeling, vol. 37, pp. 1147-1162, 2013. DOI: 10.1016/j.apm.2012.03.043

[32] P.J Pawar and R.V. Rao, "Parameter optimization of machining processes using teaching-learning-based optimization algorithm," The International Journal of Advanced Manufacturing Technology, Springer-Verlag, vol. 67, pp.995-1006, 2013. DOI: 10.1007/s00170-0124524-2

[33] R.V. Rao and V.D. Kalyankar, Parameters optimization of advanced machining processes using TLBO algorithm, The International Journal of Advanced Manufacturing Technology 67 (5-8) July 2013, 995-1006. [Online]. http://dx.doi.org/10.1007/s00170-013-4961-6.

[34] R.V. Rao, et al, "Teaching-learning-based optimization algorithm for unconstrained and constrained real-parameter optimization problems," Engineering Optimization, vol. 44, pp. 1-16, 2012. DOI: 10.1080/0305215X.2011.652103

[35] T. Niknam, et al, " $\theta$-Multiobjective Teaching-LearningBased Optimization for Dynamic Economic Emission Dispatch," IEEE Systems Journal, vol. 6, pp. 341-352. 2012 DOI: 10.1109/JSYST.2012.2183276

\section{Authors' Profiles}

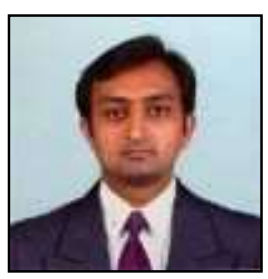

A. J. Umbarkar is presently working as an Assistant Professor in Information Technology at Walchand College of Engineering, Sangli, MS, India. He has received his Bachelor of Engineering (BE) in Computer Engineering from PICT, Pune, MS, India and his Master of Engineering (ME) from Computer Science and Engineering (CSE) from Walchand College of Engineering, Sangli, MS, India.

He has 12 years of teaching experience at UG and 6 years at PG. His research interests include Parallel Genetic Algorithms, Parallel Evolutionary Algorithms and Parallel programming. He has published about 16 research papers in Conferences and Journals.

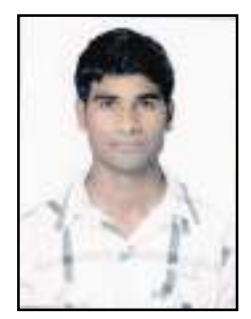

N.M.Rothe is presently working as Software Engineer in TCS. He has completed his B.Tech in Computer Engineering from Government College of Engineering, Amravati, MS, India and his M.Tech from Computer Science and Engineering (CSE) from Walchand College of Engineering, Sangli, MS, India.

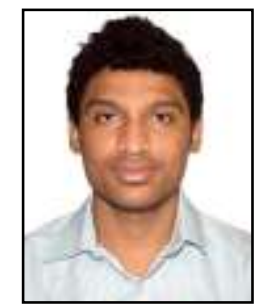

A.S.Sathe has completed Bachelor of Engineering (BE) from Pune Vidyarthi Grihas COET-Pune, MS, India. He has Industrial Experience of 6 months. He is currently Pursuing M.Tech in Information Technology at Walchand College of Engineering, Sangli, MS, India.

How to cite this paper: A. J. Umbarkar, N. M. Rothe, A.S. Sathe,"OpenMP Teaching-Learning Based Optimization Algorithm over Multi-Core System", International Journal of Intelligent Systems and Applications (IJISA), vol.7, no.7, pp.57-65, 2015. DOI: 10.5815/ijisa.2015.07.08 